Int. J. Dev. Biol. 62: 325-333 (2018)

doi: $10.1387 / \mathrm{ijdb} .170260 \mathrm{RL}$

\title{
Pou3f transcription factor expression during embryonic development highlights distinct pou $3 f 3$ and pou3f4 localization in the Xenopus laevis kidney
}

\author{
CAMILLE COSSE-ETCHEPARE ${ }^{1}$, ISABELLE GERVI ${ }^{1}$, ISABELLE BUISSON ${ }^{1}$, LAURENT FORMERY², \\ MICHAEL SCHUBERT ${ }^{2}$, JEAN-FRANÇOIS RIOU ${ }^{1}$, MURIEL UMBHAUER ${ }^{*, 1}$ and RONAN LE BOUFFANT ${ }^{*, 1}$ \\ ${ }^{1}$ Sorbonne Université, CNRS Biologie du Développement, Institut de Biologie Paris-Seine (IBPS), Paris and \\ ${ }^{2}$ Sorbonne Université, CNRS, Laboratoire de Biologie du Développement de Villefranche-sur-Mer, \\ Observatoire Océanologique de Villefranche-sur-Mer, Villefranche-sur-Mer, France
}

\begin{abstract}
The POU (Pit-Oct-Unc) genes encode a large transcription factor family comprising 6 classes (pou $1 \mathrm{fto}$ pou6f) involved in many developmental processes, such as cell commitment and differentiation. The pou3f class contains four members (pou3f1, pou3f2, pou3f3, pou3f4) characterized by expression in ectodermal tissue derivatives, such as nervous system and otic vesicle, during mammalian development. In order to obtain insights into the potential conservation of this class of transcription factors in vertebrates, we carried out a phylogenetic analysis and a comprehensive comparative study of pou3f expression in the frog Xenopus laevis. All vertebrates examined possessed members of the four pou3f subfamilies, excepting the zebrafish, which lacked a pou3f4 gene. Whole mount in situ hybridization and real-time quantitative polymerase chain reaction (RT-qPCR) analyses revealed that Xenopus pou3f genes were expressed in the forming neural tube and their expression was maintained in the brain, mostly in the dorsal part, at tailbud stages. The pou3f2, pou3f3, and pou3f4 genes were also expressed in the developing otic vesicle, and pou3f1 in some cells of the epidermis. Besides ectodermal derivatives, pou $3 f 3$ and pou $3 f 4$ were expressed in the developing kidney. Their expression started at the early tailbud stage in the pronephric anlage and partly overlapped. In the mature pronephric tubule, pou3f3 was restricted to the intermediate tubule, while pou3f4 was also expressed in the distal and connecting tubule. Together, our results highlight a significant conservation of pou3f gene expression in vertebrates and indicate that they may have distinct but also redundant functions during neural and renal development.
\end{abstract}

KEY WORDS: Pou3f, embryo, kidney, Xenopus laevis

Transcription factors serve critical roles in cell commitment and subsequent differentiation during organ formation. The POU (PitOct-Unc)genes encode a large family of transcription factors known for their roles during development (Ryan and Rosenfeld, 1997). They are defined by the presence of a highly conserved bipartite DNA-binding domain, the POU-domain, consisting of an approximately 75 amino acid N-terminal POU-specific domain and a 60 amino acid C-terminal POU-homeodomain. These two subdomains are tethered by a linker of variable length (14-26 amino acids) (Ryan and Rosenfeld, 1997). The acronym POU is derived from the names of the first genes in which the POU-domain has been discovered, i.e. the three mammalian transcription factors Pit-1, Oct-1/2, and the Caenorhabditis elegans developmental regulator unc86. The POU-domain proteins have since been described in a wide variety of animal species, but have not yet been identified in fungi or plants. In mammals, they have been grouped into six classes (Pou1f to Pou6f), depending on the amino acid sequence of their

Abbreviations used in this paper: POU, Pit-Oct-Unc; RT-qPCR, real-time quantitative polymerase chain reaction.

\footnotetext{
*Address correspondence to: Muriel Umbhauer - (iD https://orcid.org/0000-0001-7419-8070 and Ronan Le Bouffant - (iD) https://orcid.org/0000-0002-0995-7373 Sorbonne Université, CNRS-Biologie du Développement, Institut de Biologie Paris-Seine (IBPS), 9 quai Saint-Bernard, 75005 Paris, France. Tel: +33 144272901. E-mails: ronan.le_bouffant@upmc.fr-muriel.umbhauer@upmc.fr
}

Supplementary Material (3 figures +3 tables) for this paper is available at: http://dx.doi.org/10.1387/ijdb.170260RL 
POU-domain and the conservation of the variable linker region. The Pou3f class is composed of 4 intronless genes, pou3f1 (alternatively called oct6, otf6, pou50, scip, test1, tst1, nrl-22 or XLPOU1), pou3f2 (alternatively called BRN2, OCT7, OTF7, OTF-7, POUF3, brn-2, oct-7 or N-Oct3), pou3f3 (alternatively called BRN1, OTF8, oct-8 or brain-1), and pou3f4 (alternatively called BRAIN-4, BRN-4, BRN4, DFN3, DFNX2, OCT-9, OTF-9 or OTF9). They have been shown to be involved in neural and ectodermal development in mammals. Pou3f1 promotes neural fate commitment during mouse gastrulation (Zhu et al., 2014). It is implicated in Schwann cell myelinization and oligodendrocyte as well as keratinocyte differentiation (Zhao, 2013). Pou3f2 is a regulator of melanocyte growth and tumorigenesis. It is responsive to MAPK pathway activation and modulates the levels of the transcription factor MITF, hence preventing melanocytic differentiation ultimately leading to tumor metastasis (Cook and Sturm, 2008). Pou3f4 is implicated in neuron and otic vesicle development, where it controls cochlea formation. In humans, defects in POU3F4 cause X-linked deafness type 3 (Zhao, 2013). Pou3f transcription factors can act redundantly during embryonic development. In single Pou3f2 or Pou3f3 mouse mutants, no developmental defects are observable in the neocortex where these genes are co-expressed, while the double mutants of both Pou3f2 and Pou3f3 are characterized by abnormal formation of the neocortex with dramatically reduced production of layer II-IV neurons and defective migration of neurons (Cook and Sturm, 2008).
The expression of pou3f genes is documented principally in the vertebrate nervous system, but data appear fragmented. An exhaustive spatial and temporal expression survey for all pou3f genes is clearly missing in the literature. We therefore performed this analysis using the frog Xenopus laevis as a model and first assessed the conservation, phylogeny, and synteny of vertebrate pou3f class genes. We then described the expression patterns of each pou3 $f$ gene during $X$. laevis embryonic development. Our results highlight a high level of conservation of pou3f class genes across vertebrates. In $X$. laevis, partially overlapping expression in the developing neural tube suggests a potentially conserved function during vertebrate brain development. We also find that pou3f3 and pou3f4 genes are partially co-expressed in the developing pronephros, a first in any vertebrate.

\section{Results}

\section{Evolutionary conservation of Pou3f class members}

In order to characterize the evolution of Pou3f class members in vertebrates, we first analyzed alignments of the amino acid sequences of selected Pou3f proteins (Figs. 1; S1). In X. laevis, each Pou3f gene has two homologous copies ( $L$ and $S)$, whose mRNA coding sequences and protein sequences are highly conserved (more than $99 \%$ of identity). We therefore performed the alignments with only one homolog. Alignment of the four $X$. laevis Pou3f proteins (Pou3f1, Pou3f2, Pou3f3, Pou3f4) revealed a particularly high degree of sequence conservation in the POU-specific

\section{A}

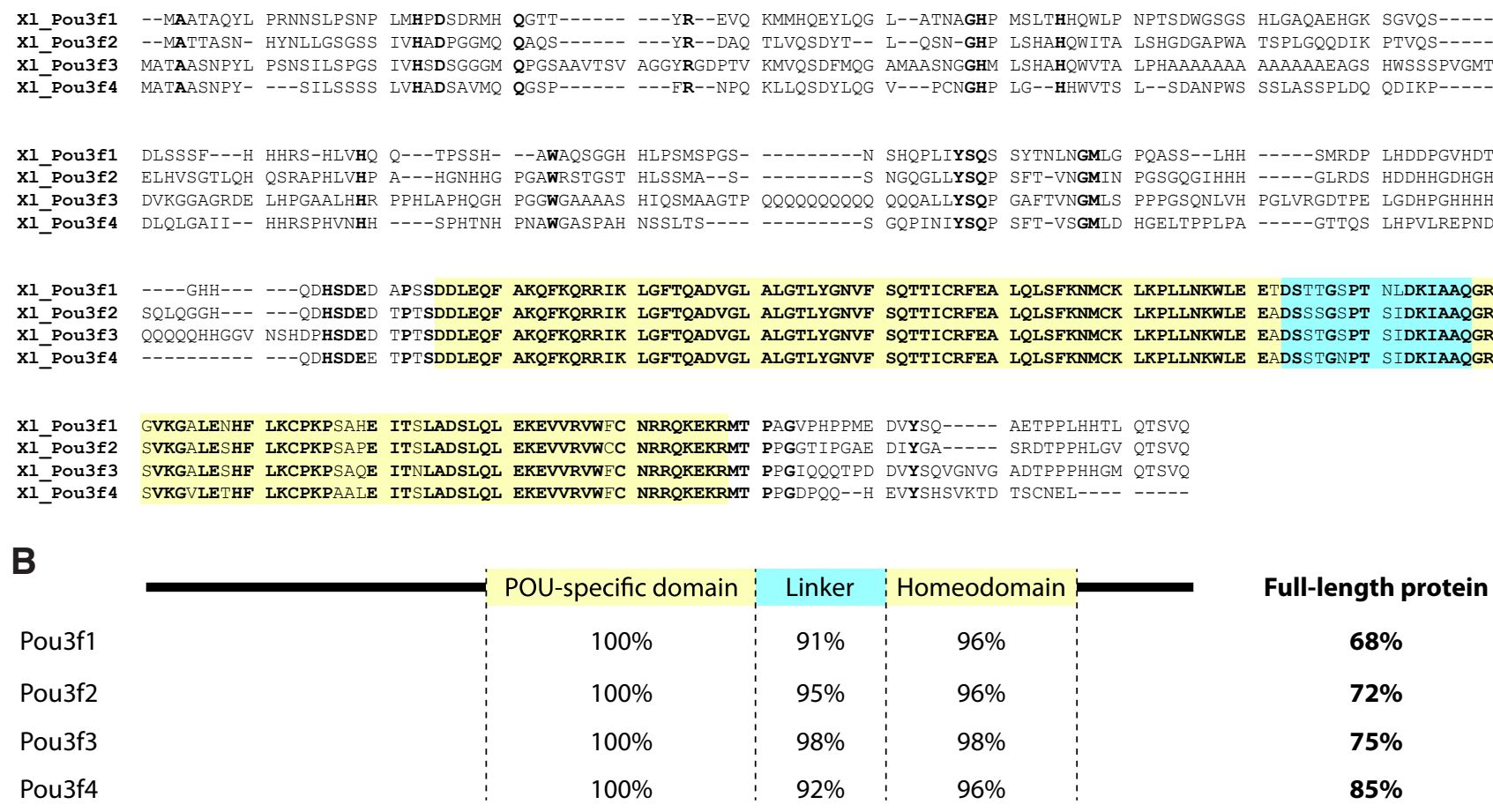

Fig. 1. Sequence analysis of vertebrate Pou3f proteins. (A) Sequence alignments of the Xenopus laevis (XI) Pou3f1, Pou3f2, Pou3f3, and Pou3f4 proteins. Conserved residues are shown in bold, the POU-specific domain and the POU-homeodomain are highlighted in yellow, and the linker region is indicated in cyan. (B) The percentage of sequence identity for each vertebrate Pou3f subfamily (Pou3f1, Pou3f2, Pou3f3, and Pou3f4) relative to the Xenopus laevis sequence is indicated for the POU-specific domain, the linker, the POU-homedomain as well as for the whole (i.e. full-length) protein. The percentages are the average sequence identities of the human (Homo sapiens), mouse (Mus musculus), chicken (Gallus gallus), and spotted gar (Lepisosteus oculatus) sequences relative to the African clawed frog (Xenopus laevis) sequence. 


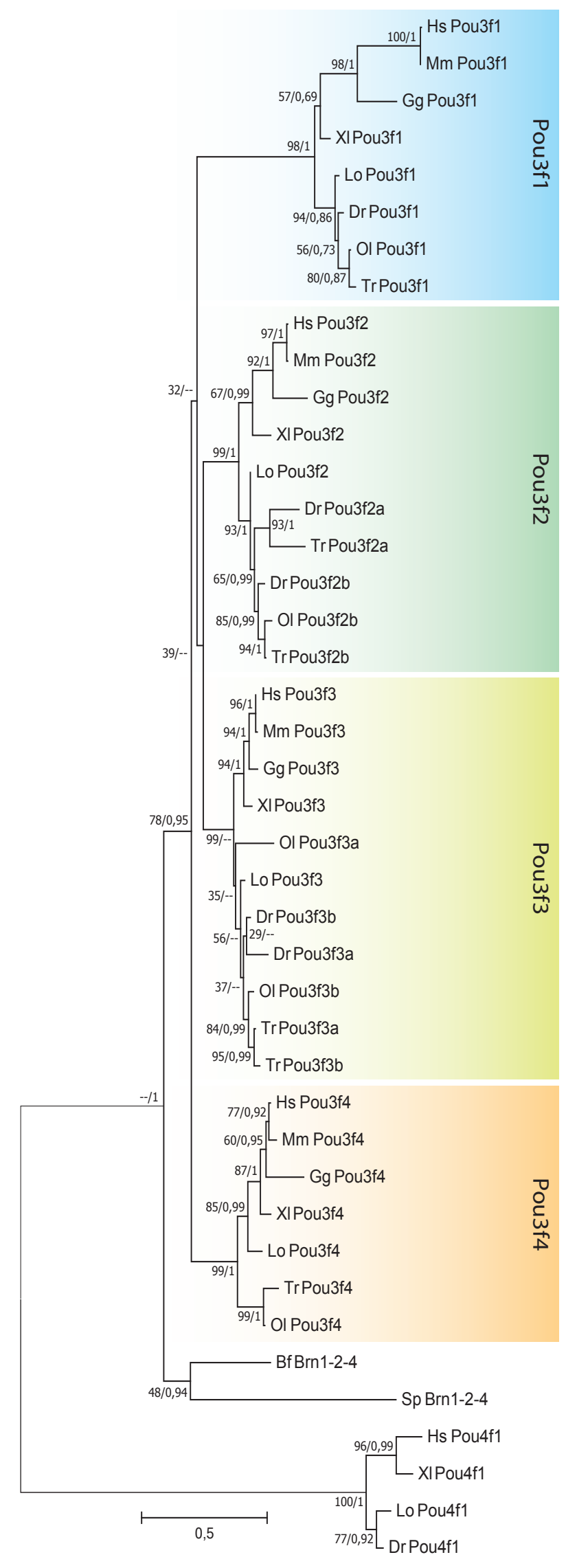

domain, the linker region as well as the POU-homeodomain (Fig. 1 A). Comparisons of the four $X$. laevis Pou3f class proteins with their respective orthologs from human, mouse, chicken, and the spotted gar further supported this finding, with sequence identities ranging between $68 \%$ and $85 \%$ for the full-length proteins, but reaching $100 \%$ within the POU-specific domain, between $91 \%$ and $98 \%$ in the linker region, and between $96 \%$ and $98 \%$ in the POU-homeodomain (Fig. 1B). To assess the evolutionary diversification of pou3f class genes in vertebrates, we next performed phylogenetic analyses of Pou3f proteins (Figs. 2, $\mathrm{S} 1)$. The resulting phylogenetic tree revealed that the vertebrate pou3 $f$ genes encoding these proteins group into four subfamilies (pou3f1, pou3f2, pou3f3, pou3f4), with each vertebrate analyzed possessing at least one member of each of these subfamilies (Fig. 2), except for the zebrafish that lacks a pou3f4 gene. The phylogeny further revealed that the genomes of teleost fish encode additional paralogs in the pou3f2 and pou3f3 subfamilies and that some fish lineages might have independently lost specific poußf genes, which seems to be the case for zebrafish pou3f4 as well as for medaka pou3f2a (Fig. 2). Of note, non-vertebrates seem to generally possess only a single pou3f gene (Fig. 2). To further investigate the evolution of pou3f genes in vertebrates, we next carried out a synteny analysis of the pou3f loci of several vertebrate species (Fig. 3). This analysis largely supported the results obtained by the phylogenetic tree reconstruction in that, while vertebrate genomes are generally characterized by at least four pou3f genes, the genomes of teleost fish have experienced both additional large-scale duplications (resulting in additional teleost pou3f2 and pou3f3 paralogs) and species-specific pou3f gene losses (leading to the absence of zebrafish pou3f4 and medaka pou3f2a) (Fig. 3).

\section{Spatial and temporal expression of pou3f1 during X. laevis development}

Temporal and spatial expression of pou3f1 during $X$. laevis embryonic development was examined by real-time quantitative polymerase chain reaction (RT-qPCR) and whole mount in situ hybridization. Pou3f1 expression begins at gastrulation (st 10), increases during neurulation (st 13-16) and tailbud stages (st 22) to reach a maximum level at the late tailbud stage (st 28 ), with its expression subsequently decreasing at the tadpole stage (st 37 ) (Fig. 4A). By in situ hybridization, the signal is first detected in the marginal zone during gastrulation (Fig. 4B, a). At the mid-neurula

Fig. 2. Phylogenetic relationship of vertebrate Pou3f proteins. Phylogenetic tree of the Pou3f family based on sequences from human (Homo sapiens, Hs), mouse (Mus musculus, Mm), chicken (Gallus gallus, Gg), African clawed frog (Xenopus laevis, XI), zebrafish (Danio rerio, Dr), medaka (Oryzias latipes, Ol), Japanese puffer (Takifugu rubripes, Tr), spotted gar (Lepisosteus oculatus, Lo), Florida amphioxus (Branchiostoma floridae, Bf), and the purple sea urchin (Strongylocentrotus purpuratus, Sp). The Pou3f1, Pou3f2, Pou3f3, and Pou3f4 subfamilies are respectively highlighted in blue, green, yellow, and orange. The tree was rooted with members of the Pou4f1 subfamily. Although trees were calculated using both the Maximum Likelihood (ML) and Bayesian Inference (BI) methods, only the $M L$ tree is shown, with branch lengths being representative of sequence substitution rates. Branch support is indicated as bootstrap percentages from the $M L$ analysis (ranging from 0 to 100) / posterior probabilities (ranging from 0 to 1) from the BI analysis. "--" indicates that the branching patterns of the $M L$ and $B /$ analyses diverged at this node and that one of the scores is thus unavailable. 

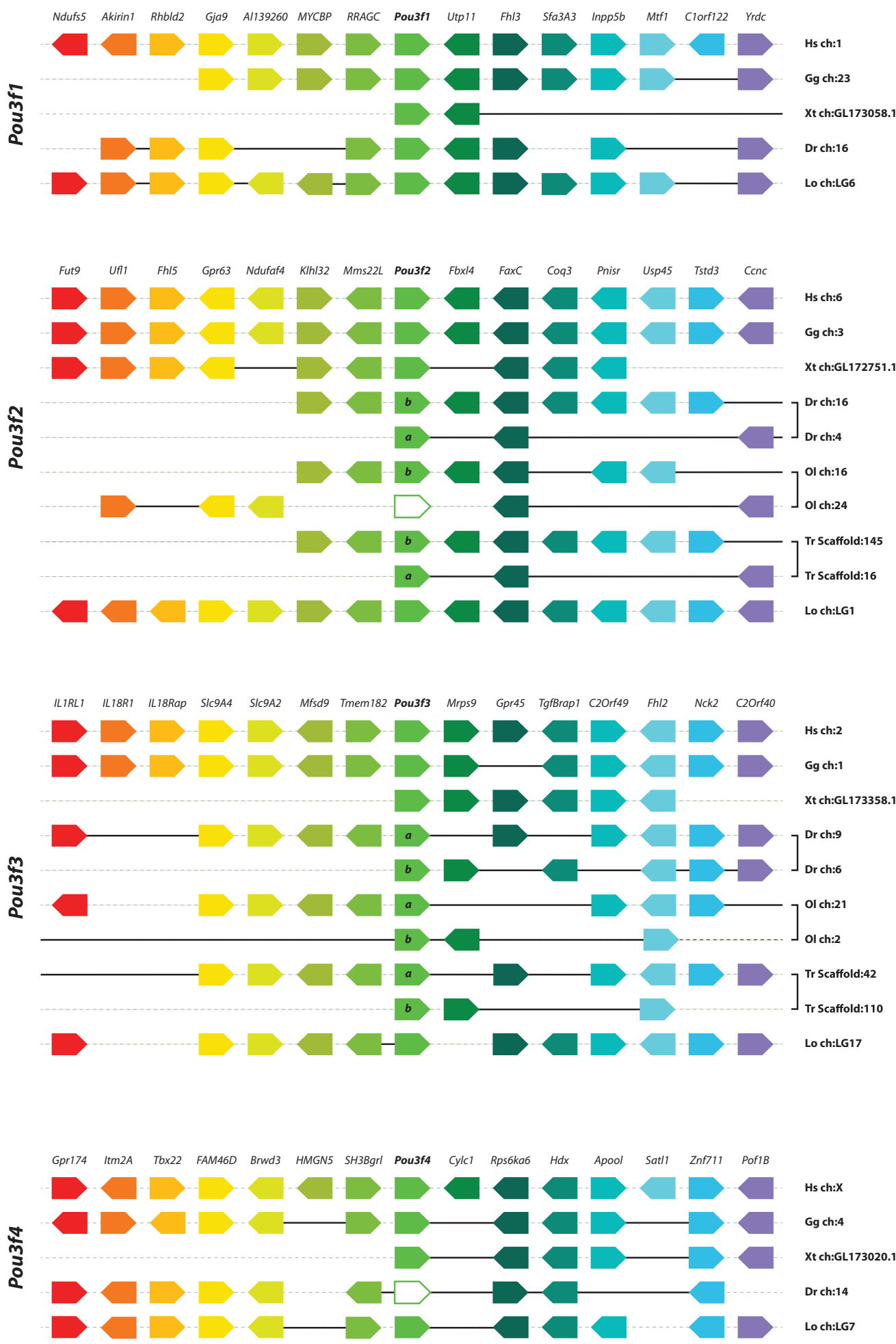

Fig. 3. Genomic organization of vertebrate pou3f loci. Genomic organization and gene synteny comparisons of vertebrate pou3f1, pou3f2, pou3f3, and pou3f4 genes. Dotted lines represent chromosome loci in the genomes of human (Homo sapiens, Hs), chicken (Gallus gallus, Gg), Western clawed frogs Xenopus tropicalus, Xt), zebrafish (Danio rerio, Dr), medaka (Oryzias latipes, Ol), Japanese puffers (Takifugu rubripes, Tr), and spotted gars (Lepisosteus oculatus, Lo). Genes are shown as colored pentagon arrows, with the transcriptional orientation of a given gene locus being indicated by the direction of the arrow. When a pou3f paralog is not found in a given species, the pentagon is white. Gene loci are named according to the nomenclature used in human and orthologs in the different species are shown in identical colors. Gene paralogs are indicated by letters. Bold black lines between polygons mark changes in genetic linkage and hence in gene synteny relative to the situation in the human genome. stage (st 16), pou3f1 is expressed in the anterior neural folds (Fig. 4B, c), and its expression is maintained in the lateral part of the neural tube all along its antero-posterior axis at stage 23 (Fig. 4B, d, e). Subsequently, pou3f1 is expressed in the dorsal half of the brain excepting the most dorsal part at late tailbud and tadpole stages (Fig. 4B, f-i). Transcripts of pou3f1 are also found in the optic vesicle at the early tailbud stage (Fig. 4B, d) and in the retina at later stages (Fig. 4B, f-i). In addition, from the early tailbud stage onward, there is a strong dotted staining of pou3f1 in the epidermis, and, from the mid-neurula stage, pou3f1 expression is further detectable in neuro-ectodermal tissues. This spatial expression pattern was confirmed by RT-qPCR analysis of different embryonic regions: these results clearly showed a relative enrichment of pou3f1 transcripts in the dorsal half of the embryo as well as in the head (Fig. 4C).

\section{Spatial and temporal expression of pou3f2 during $\mathrm{X}$. laevis devel- opment}

RT-qPCR analysis revealed a high variability of pou3f2 expression levels, likely due to the very dynamic expression pattern of pou3f2 (data not shown). Expression is not detectable by whole mount in situ hybridization before the early neurula stage when it is detectable in the anterior part of the neural plate as a stripe in the neural folds (Fig. 5A, a,b). Its neural expression is restricted to the brain during tailbud and tadpole stages (Fig. 5A, C-i). Examination of transverse sections at the early tailbud stage (st 23) highlights expression in the dorso-lateral part of the hindbrain (Fig. 5A, d). The pou3f2 gene is also expressed in the otic vesicle at late tailbud stages (st 29/30-39) (Fig. 5A, e,f,h,i) and in two cell populations ventral to the eye that might be neural crest cell derivatives (arrowhead) (Fig. 5A, f,i). This spatial expression pattern was confirmed by RT-qPCR analysis of different embryonic regions, showing a strong relative enrichment of pou3f2 transcripts in the head (Fig. $5 B)$. 
A

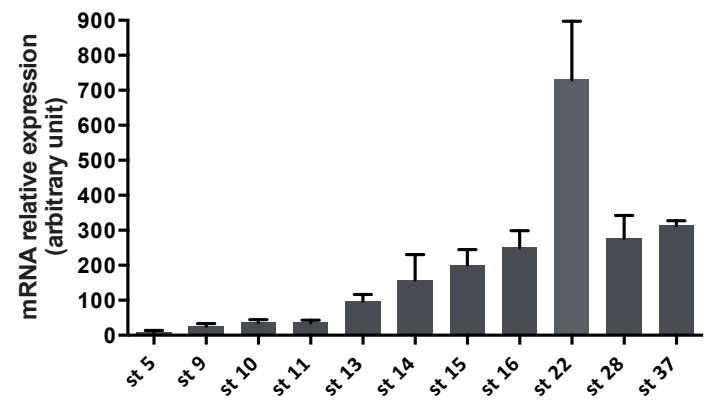

B

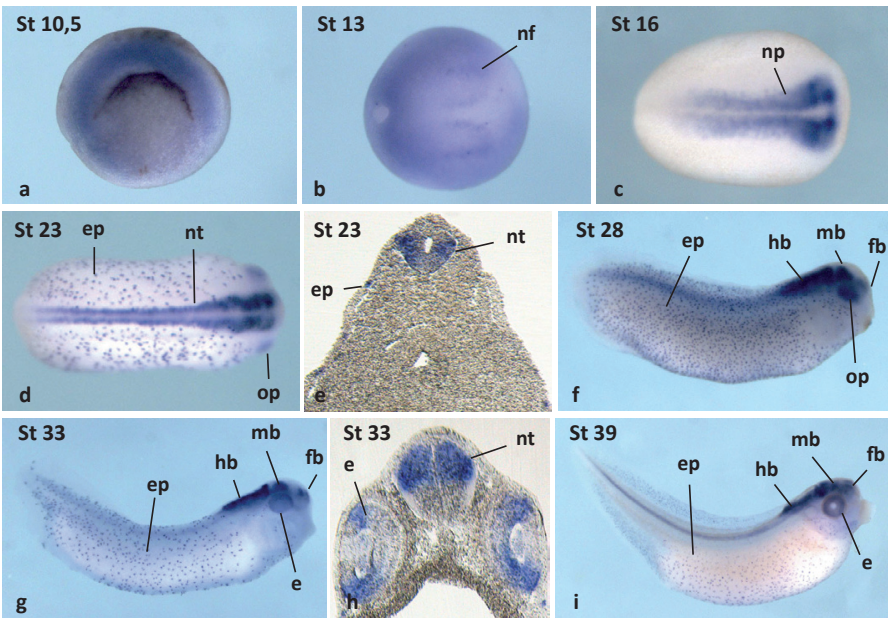

C

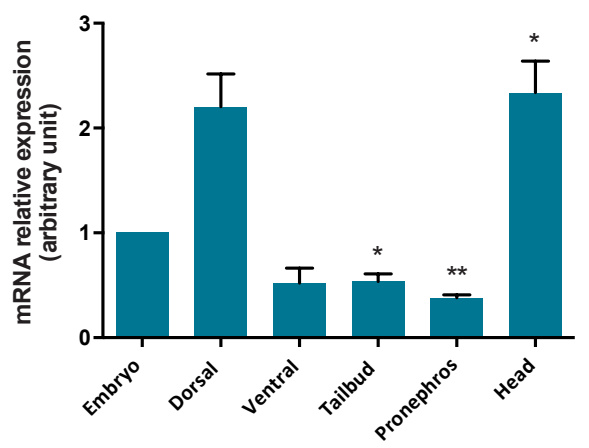

\section{Spatial and temporal expression of pou3f3 during $\mathrm{X}$. laevis development}

RT-qPCR detected no maternal pou3f3 expression and very low levels of expression during gastrulation. Pou3f3 expression increases slowly during neurulation (st 13-16) and during tailbud stages (st 22 and 28), before decreasing at the tadpole stage (st 37) (Fig 6A). Pou3f3 transcripts were first detected by in situ hybridization in the anterior neural plate as a stripe in the neural folds (Fig. 6B, a,b). From the early tailbud stage, pou3f3is strongly

\section{A}

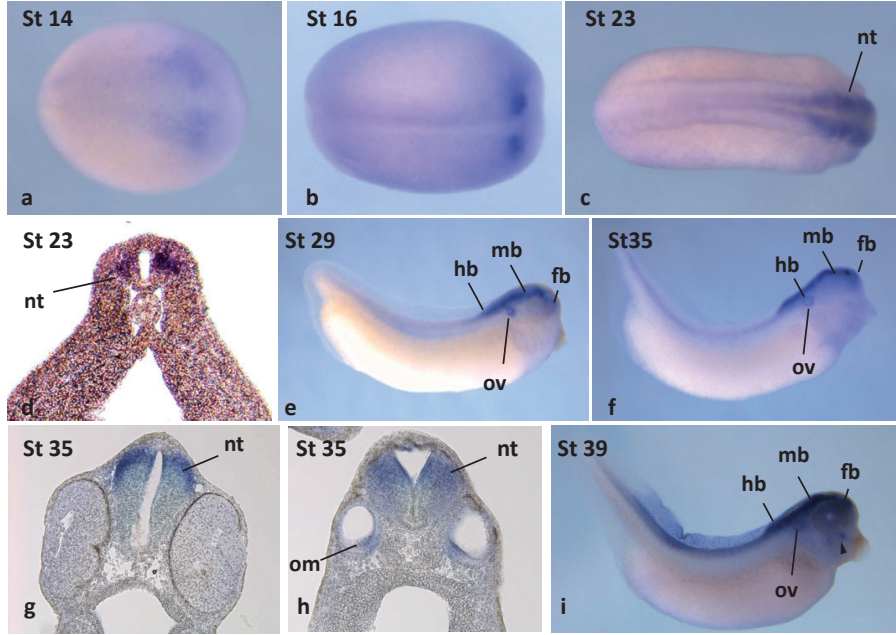

B

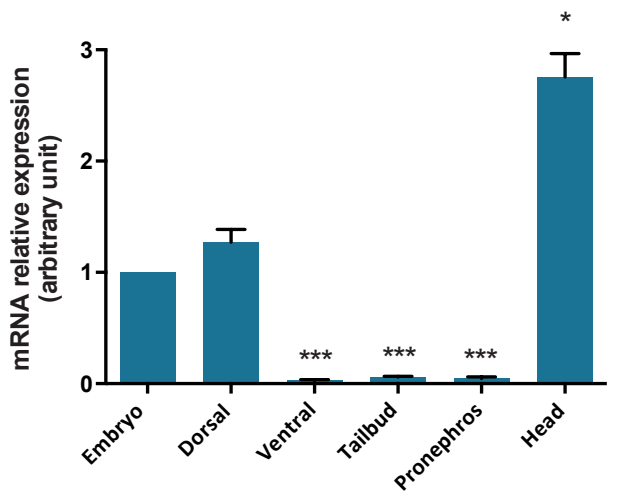

Fig. 4 (left). Spatio-temporal expression of pou3f1 during Xenopus laevis development. (A) Expression of pou3f1 analyzed by real-time quantitative polymerase chain reaction (RT-qPCR) at indicated stages of development. Average values from three independent experiments. (B) Whole mount in situ hybridization for pou3f1 at indicated stages of development. Early gastrula, vegetal view (a). Neurula, dorsal view (b,c). Tailbud, dorsal (d) and lateral (f) view. Tadpole, lateral view (g,i). Transverse sections at the level of the developing brain at tailbud stage (e) and at the level of the developing eye at tadpole stage (h). Abbreviations: e, eye; ep, epidermis; fb, forebrain; hb, hindbrain; mb, midbrain; nf, neural fold; np, neural plate; $n t$, neural tube; op, optic placode. (C) Expression of pou3f1 analyzed by RT-qPCR on dissected explants from tailbud-stage embryos, stage 25. Embryos were dissected either into ventral and dorsal halves or into tailbud, pronephros and head. Average values from three independent experiments. ${ }^{*}: P<0,05$; **: $P<0,005$, relative to the whole embryo.

Fig. 5 (right). Spatio-temporal expression of pou3f2 during Xenopus laevis development. (A) Whole mount in situ hybridization for pou3f2 at indicated stages of development. Neurula, dorsal view (a,b). Tailbud, dorsal (c) and lateral (e) view. Tadpole, lateral view (f,i). Transverse sections at the level of the developing brain $(d, g, h)$, and otic vesicle (h). Abbreviations: (fb) forebrain, (hb) hindbrain, (mb) midbrain, (nt) neural tube, (om) otic mesenchyme, (ov) otic vesicle. (B) Expression of pou3f2 analyzed by real-time quantitative polymerase chain reaction (RT-qPCR) on dissected explants from tailbud embryos (stage 25). Embryos were dissected either into ventral and dorsal halves or into tailbud, pronephros and head. *: $P<0,05 ;{ }^{* * *}: P<$ 0,001 , relative to the whole embryo. 
A

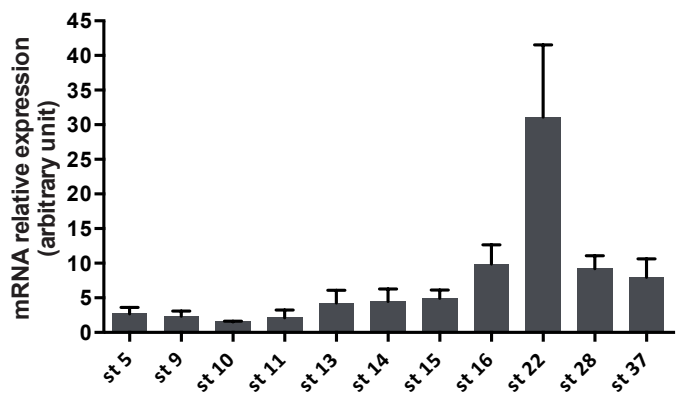

B

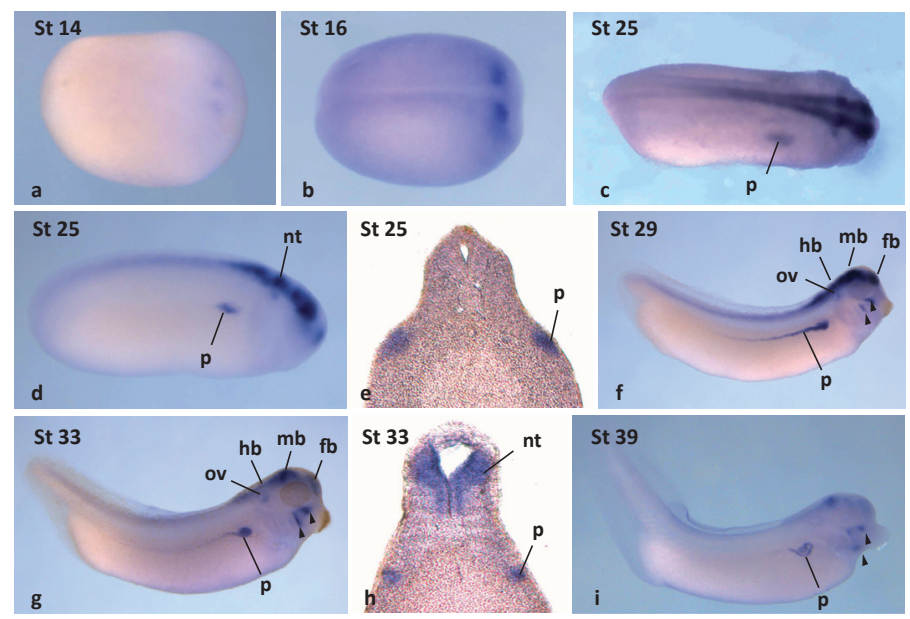

C

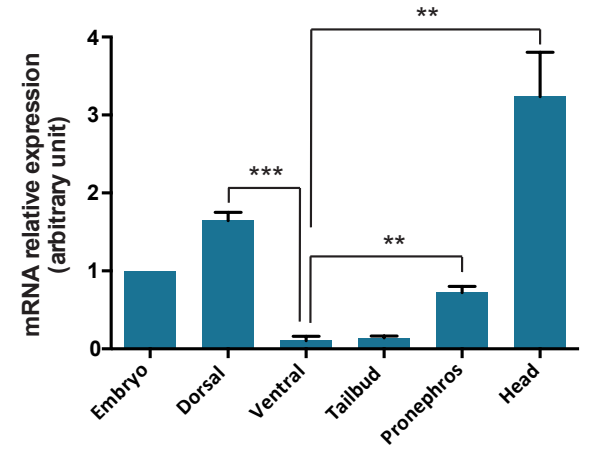

A

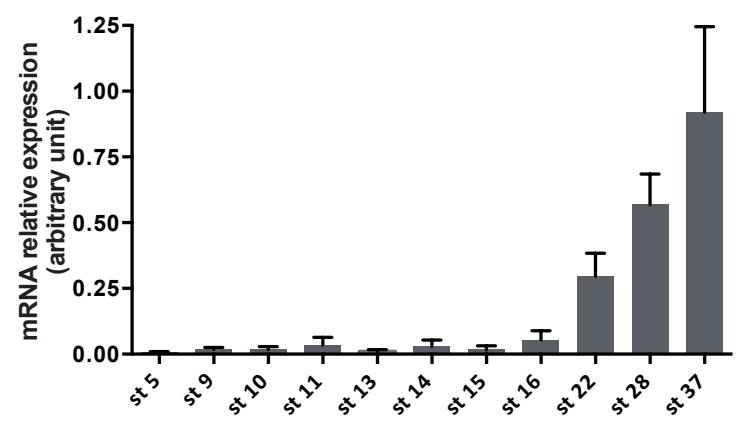

B

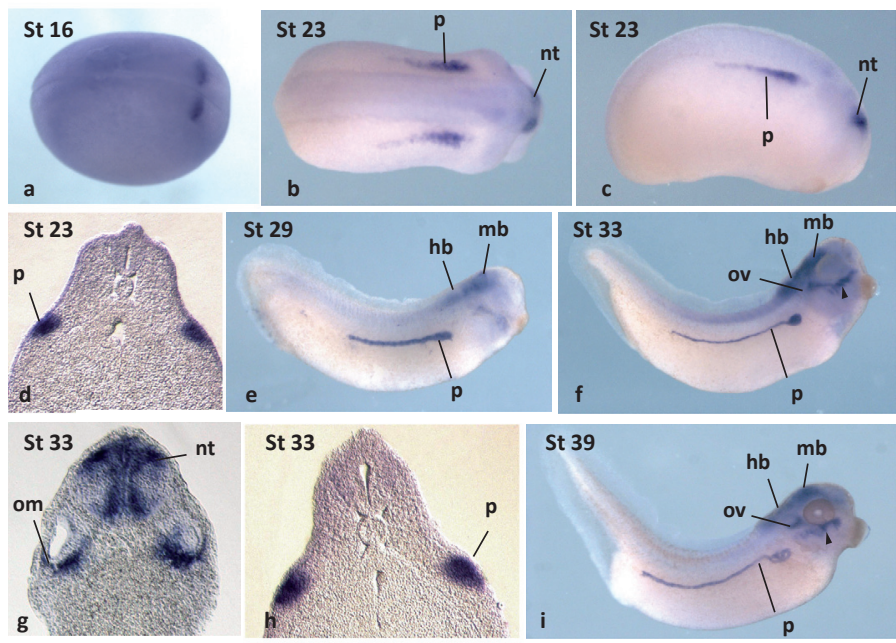

C

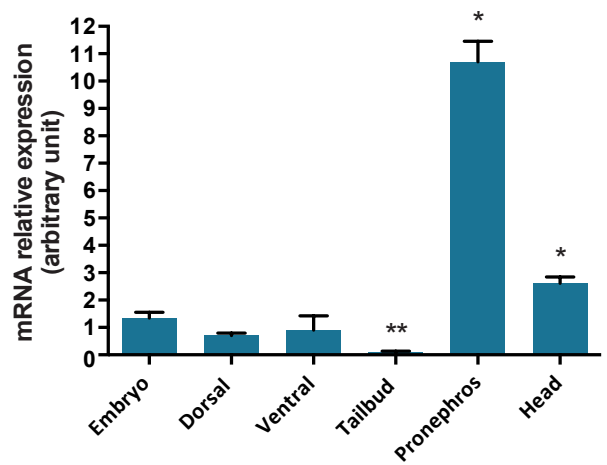

Fig. 6 (left). Spatio-temporal expression of pou3f3 during Xenopus laevis development. (A) Expression of pou3f3 analyzed by real-time quantitative polymerase chain reaction (RT-qPCR) at indicated stages of development. (B) Whole mount in situ hybridization for pou3f3 at indicated stages of development. Neurula, dorsal view (a,b). Tailbud, dorsal $(c)$ and lateral $(d, f)$ view. Tadpole, lateral view (g, i). Transverse sections at the level of the developing pronephros (e,h). Abbreviations: (fb) forebrain, (hb) hindbrain, (mb) midbrain, (nt) neural tube, (ov) otic vesicle, (p) pronephros. (C) Expression of pou3f3 analyzed by RT-qPCR on dissected explants from tailbud-stage embryos (stage 25). Embryos were dissected either into ventral and dorsal halves or into tail bud, pronephros and head. ${ }^{*}: P<0,005 ;{ }^{*}: P<0,001$, relative to the ventral half.

Fig. 7 (right). Spatio-temporal expression of pou3f4 during Xenopus laevis development. (A) Expression of pou3f4 analyzed by real-time quantitative polymerase chain reaction (RT-qPCR) at indicated stages of development. Average values from three independent experiments. (B) Whole mount in situ hybridization for pou3f4 at indicated stages of development. Neurula, dorsal view (a). Tailbud, dorsal (b) and lateral view (c,e). Tadpole, lateral (f,i) view. Transverse sections at the level of the developing pronephros (d, h) and otic vesicle (g). Abbreviations: (fb) forebrain, (hb) hindbrain, (mb) midbrain, (nt) neural tube, (om) otic mesenchyme, (ov) otic vesicle, (p) pronephros. (C) Expression of pou3f4 analyzed by RT-qPCR on dissected explants from tailbud-stage embryos (stage 25). Embryos were dissected either into ventral and dorsal halves or into tail bud, pronephros and head. *: $P<0,05 ;{ }^{*}$ : $P<0,005$, relative to the whole embryo. 
expressed in the developing forebrain, midbrain, and hindbrain where expression is maintained at least until the tadpole stage (Fig. 6B, c-i). Outside the brain, pou3f3 is also detectable in the developing pronephros. At the early tailbud stage, transcripts are present in the proximal part of the pronephric anlage (Fig. $6 \mathrm{~B}, \mathrm{~d}, \mathrm{e})$. Pronephric expression of pou3f3 persists in the developing tubule during tailbud and tadpole stages (Fig. 6B, f-i). Pou3f3 is also expressed in the otic vesicle and possibly in anterior neural crest cell derivatives (arrowhead) (Fig. 6B, f,g,i). RT-qPCR analysis of different regions of early tailbud stage embryos confirmed these observations, with strong relative expression of pou3f3 in the head and pronephros (Fig. 6C, S2).

\section{Spatial and temporal expression of pou3f4 during $\mathrm{X}$. laevis development}

Pou3f4starts to be weakly expressed in the embryo at the mid-neurula stage (st 16), and its expression gradually increases during tailbud (st 22 and 28) and tadpole stages (st 37), as shown by RT-qPCR (Fig. 7A). Using whole mount in situ hybridization, pou3f4 transcripts were first detectable at the mid-neurula stage in the anterior part of the neural folds (Fig. 7B, a). At tailbud stages, pou3f4 expression is conspicuous in the developing midbrain and hindbrain where it is maintained at tadpole stages (Fig. 7B, e,f,g,i). Apart from the brain, pou3f4 is also strongly expressed in the pronephric anlage at the early tailbud stage (Fig. 7B, b-d), with expression persisting in the developing pronephric tubule (Fig. 7B, e,f,h,i). In addition, pou3f4 transcripts are detectable in groups of cells surrounding the eye (arrow head) (Fig. 7B, f,i) as well as in the condensing otic mesenchyme, which lies ventral to the otic vesicle (Fig. 7B, g). RT-qPCR analysis performed on different embryonic regions confirmed that pou3f4 is strongly expressed in the head and the pronephric anlage (Fig. 7C).

\section{Differential expression of pou3f3 and pou $3 \mathrm{f} 4$ in the developing $\mathrm{X}$. laevis pronephros}

In order to define more precisely the expression domains of pou3f3 and pou3f4 in the developing $X$. laevis pronephros, we performed double staining by whole mount in situ hybridization. We compared pou3f3 and pou3f4 expression with that of pax8, which marks the entire presumptive pronephric region at the early and mid-tailbud stages (Carroll and Vize, 1996). The expression of pou3f3 is restricted to the most anterior part of the pronephric anlage (Fig. 8A, d,e), whereas pou3f4 is expressed in the entire pronephric anlage at the exception of the most dorso-anterior part (Fig. 8A, $\mathrm{h}, \mathrm{i})$. Transverse sections confirm these observations and reveal that pou3f3 and pou3f4 expression within the pronephric mesoderm is restricted to the somatic layer that gives rise to the pronephric tubule. No expression is detected in the splanchnic layer that will form the glomus (Fig. 8A, f,g,j,k). At the tadpole stage, the pronephric tubule is segmented along the proximo-distal axis into proximal, intermediate, distal, and connecting tubule (Raciti et al., 2008). In contrast to pax8, neither

A

B

C pou3f3 nor pou $3 f 4$ is expressed in the proximal tubule (Fig. 8B). The pou3f3 signal is in the intermediate tubule and the distal tubule, but expression in the latter declines as maturation of the tubule progresses (Fig. 8B, d-f) (Fig. 6B, f-i). The pou3f4 gene is
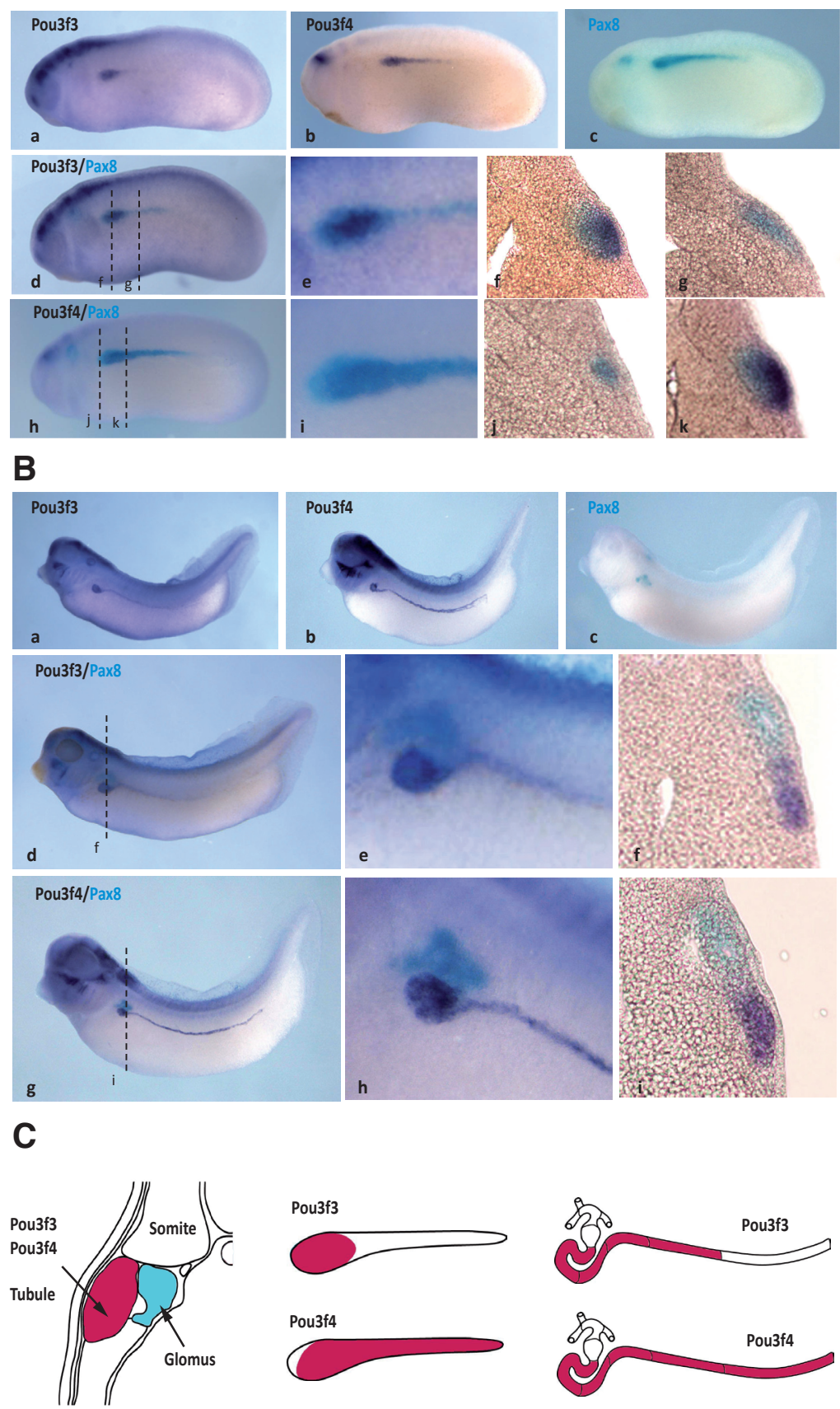

Fig. 8. Differential pronephric expression of pouf3 and pou3f4 in Xenopus laevis. Whole mount in situ hybridization analysis of pronephric expression of pou $3 \mathrm{f} 3$ and pou3f4 (dark blue) compared to pax8 (light blue) in tailbud-stage embryos (stage 25) (A) and in the differentiated tubule in late tailbud-stage embryos (st 35) (B). Transverse sections in the anterior (A.f,j) and posterior part (A.g, k) of the anlage, and in the anterior part of the tubule (B.f,i). (C) Schematic representation ofpou3f3 and pou3f4 expression in the pronephros: transverse section of a tailbud-stage embryo (left), lateral view of the pronephric anlage (middle) and of the differentiated tubule (right). 
strongly expressed in the intermediate and distal tubule as well as the connecting tubule (Fig. 8B, g-i).

\section{Discussion}

The present study aims at characterizing the class $3 \mathrm{POU}$ transcription factor complement of vertebrates, with a special focus on the pou3fgenes from the frog $X$. laevis. Amino acid alignments revealed the general conservation of vertebrate Pou3f class proteins. Based on the fact that non-vertebrates generally encode only a single pou3f ortholog, the results of our phylogenetic and synteny analyses further indicate that the four vertebrate pou3 $f$ genes result from two successive duplications of a single ancestral pou3f gene in the last common ancestor of all vertebrates. The evolutionary history of the vertebrate pou3fclass is thus consistent with the hypothesis that two whole-genome duplication (WGD) events occurred during early vertebrate evolution (Van de Peer et al., 2017). The existence of two pou3f2 and pou3f3 paralogs in teleost fish further supports the scenario of an additional, third, WGD marking the evolutionary diversification of the teleost fish lineage (Van de Peer et al., 2017). Intriguingly, we also found two examples of lineage-specific pou3f gene losses in teleost fish: pou $3 f 4$ in the zebrafish and pou3f2a in the medaka. Future work will have to address, how widespread these lineage-specific pou3 $f$ gene losses are in teleost fish, whether they are directly linked to the third WGD and thus to the increased teleost fish pou3fcomplement, and whether they compensate, for example, gene dosage effects.

Our results show that all four $X$. laevis pou3f genes are expressed during neurulation in the forming neural tube and that their expression is maintained in the brain, mostly in the dorsal part, at tailbud stages. Neural expression of Xenopus pou3f3 has already been described and our results largely confirm the published data (Square et al., 2015). Our results further highlight an evolutionary conserved expression of pou $3 f$ members in neural tissues. In mice, for example, pou3f1 is expressed in the anterior neuro-ectoderm at E7.5 (Zhu et al., 2014). While the activity of this gene is subsequently required in the forebrain and midbrain for the regulation of neural commitment (Zhu et al., 2014), pou3f4 is involved in neuron differentiation (Zhao, 2013). Furthermore, pou3f2 and pou3f3 show similar expression in the nervous system and a high level of functional redundancy. They are expressed in the neocortex, primarily in neurons of layers II-IV, and the analysis of pou3f2/pou3f3 double mutants showed that both are involved in cortical neural migration, layer production, and neurogenesis (Cook and Sturm, 2008). Similarly, partial redundancy of pou3f1 and pou3f2 in Schwann cell differentiation has been described (Cook and Sturm, 2008). Interestingly, we showed that pou3f1, known to be involved in epidermal keratinocyte differentiation in mammals, is expressed in the Xenopus epidermis (Zhao, 2013). We also observed that pou3f2, pou3f3, and pou3f4 are expressed in the developing Xenopus ear, as previously described in the mouse (Mutai et al., 2009, Phippard et al., 1999). Together with the fact that pou 34 is responsible for X-linked deafness type 3 in humans (Zhao, 2013), these data suggest a conserved role for pou3f4 during vertebrate ear development. Of note, we did not find pou3f4 expression in the mesoderm during gastrulation, contrary to what has previously been published (Witta et al., 1995). Our result has been confirmed by RT-qPCR on dissected gastrulae, with no significant expression having been detected in the mesoderm compared to other tissues (Fig. S3). Given that the authors of the previous study used an in situ hybridization probe targeting the entire pou3f4 sequence (Witta et al., 1995), this full-length probe might have also recognized other pou3fgenes via the conserved POU-domain, for example pou3f1, which is highly expressed in the mesoderm during gastrulation.

Here, we show for the first time that pou3f3 and pou3f4, but neither pou3f1 nor pou3f2, are expressed in the developing Xenopus pronephros. In mice, pou $3 \mathrm{f} 3$ is expressed in the renal vesicle of the developing metanephros, and, later on, its expression is restricted to Henle's loop and to the distal convoluted tubule. The pou3f3 gene has been shown to play a crucial role in the development of distinct nephron segments: pou3f3 knock-out mice exhibit impaired elongation and differentiation of the developing Henle's loop and perinatal death due to renal failure (Nakai et al., 2003). More recently, analysis of the mouse mutant Pou $3 \mathrm{f}^{\llcorner 423 P}{ }^{2}$, characterized by a point mutation that leads to the alteration of a specific amino acid affecting the conserved homeobox domain of the protein, further revealed an involvement of pou3f3 in the regulation of the overall number of developing nephrons (Rieger et al., 2016). Our results suggest a conserved role for pou3f3 during vertebrate kidney development, as, in Xenopus, pou3f3 is expressed in the anterior part of the pronephric anlage and later on in the intermediate and distal pronephric tubule, which is the structural and functional analog of Henle's loop (Raciti et al., 2008). We also observed that pou3f4 is expressed in the developing Xenopus pronephros, but with a slightly different pattern than pou3f3. It is therefore interesting to note that part of the pronephric anlage and some tubule segments express both pou3f3and pou3f4, suggesting a possible redundancy or synergy, whereas some pronephric structures express only pou3f3 (the most proximal part of the anlage) or pou3f4 (the most distal part of the anlage and the tubule). In mice, three independent transcriptomic analyses have established that pou3f4 is expressed in the ureteric bud, but not in the renal vesicle at E11.5 (Georgas et al., 2009, Schmidt et al., 2005, Smeeton et al., 2016), suggesting that pou3f3 and pou3f4 are also differentially expressed during metanephros development in mice. Together, these data suggest that pou3f3 and pou3f4 could have both distinct and redundant functions during kidney development in vertebrates. It will be important to perform single and double loss of function analyses in Xenopus to study the functional conservation of these genes during vertebrate kidney development.

\section{Materials and Methods}

\section{Sequence alignments, phylogenetic tree calculations and genomic linkage analyses}

Amino acid sequences included in the analysis were obtained by BLAST searches and are listed in Supplementary Table S1. Alignments were performed using MUSCLE as implemented in MEGA7 (Kumar et al., 2016) and then refined by eye. The final alignment included 42 amino acid sequences with a total of 317 positions (Fig. S1). Sequence identities for full-length $X$. laevis Pou3f proteins and their subdomains were calculated using a BioEdit sequence identity matrix (Hall, 1999). Phylogenetic trees were calculated with both the Maximum Likelihood (ML) and Bayesian inference (BI) methods, using a Dayhoff matrix-based substitution model with a discreet Gamma distribution (Schwarz and Dayhoff, 1979). ML tree calculations were conducted in MEGA7 (Kumar et al., 2016) with the robustness of each node being estimated by bootstrap analyses (in 1000 pseudoreplicates) (Felsenstein, 1985). BI trees were calculated using 
MrBayes version 3.1.2 (Huelsenbeck and Ronquist, 2001) in 1,000,000 generations with sampling of trees every 100 generations and a burn-in period of $25 \%$. The branching pattern of the ML tree was retained in the final tree figure, displaying, at each node, the bootstrap support of the ML analysis as well as the posterior probability support of the $\mathrm{BI}$ analysis. The tree is drawn to scale, with branch lengths indicating the number of substitutions per site. Synteny analyses were performed using Genomicus v89.02 (Louis et al., 2015) and complemented manually based on genome locus searches of the NCBI database (Altschul et al., 1990). The final synteny figure was adapted from the Genomicus output.

\section{$\mathrm{X}$. laevis embryos and dissections}

$X$. laevis were purchased from the CNRS Xenopus breeding Center (Rennes, France). Embryos were obtained after artificial fertilization and were raised in modified Barth's solution (MBS). Staging was carried out according to the normal table of $X$. laevis development (Nieuwkoop, 1967). Dissections were performed as previously described (Le Bouffant et al., 2014).

\section{Real-time quantitative PCR}

RNA extraction, reverse transcription, and real-time quantitative polymerase chain reaction (RT-qPCR) experiments were carried out as previously described (Le Bouffant et al., 2014). The Comparative Ct method was used to determine the relative quantities of mRNA, using ODC mRNA as the endogenous reporter. Identical results were obtained using $\beta$-actin mRNA as the endogenous reporter (data not shown). Each RNA sample was analyzed in duplicates. All primers were used at a final concentration of $400 \mathrm{nM}$. Sequences of the oligonucleotides used are given in Supplementary Table S2. Each data point represents the mean \pm standard error of the mean (SEM) of at least three independent experiments. Data were analyzed using $\mathrm{R}$ Commander ( $\mathrm{R}$ software) by paired Student's t-test.

\section{In situ hybridization}

Whole mount in situ hybridizations were carried out as previously reported (Le Bouffant et al., 2014). To obtain the plasmids for synthesis of antisense RNA probes, PCR products were generated from cDNA libraries using specific primers listed in Supplementary Table S3. PCR products were cloned into the pCRII vector according to manufacturer's instructions (Invitrogen), and digoxigenin-labeled antisense RNA probes were transcribed from linearized plasmids using SP6 or T7 polymerase. After in situ hybridization, embryos were sectioned at $60 \mu \mathrm{m}$ thickness using a vibratome. In situ hybridization with a sense probe was performed as a control for each antisense probe. Signal above background was never detected with the sense probes.

\section{Acknowledgements}

We want to thank Sylvie Authier, Edouard Manzoni, and Abdelkrim Mannioui for excellent technical assistance in the maintenance of the Xenopus animal facility, Aurellia Galliot for help with in situ hybridization, and Sophie Gournet for illustration work. The authors are further indebted to Philippe Dru and João E. Carvalho for supporting the bioinformatic analyses. This work was supported by grants from the CNRS and from Sorbonne Université. CCE was financed by a 2014-2017 "Contrat Doctoral" from the doctoral school "Complexité du Vivant".

\section{References}

ALTSCHUL, S.F., GISH, W., MILLER, W., MYERS, E.W. and LIPMAN, D.J. (1990). Basic local alignment search tool. J Mol Biol 215: 403-410.

CARROLL, T.J. and VIZE, P.D. (1996). Wilms' tumor suppressor gene is involved in the development of disparate kidney forms: evidence from expression in the Xenopus pronephros. Dev Dyn 206: 131-138.

COOK, A.L. and STURM, R.A. (2008). POU domain transcription factors: BRN2 as a regulator of melanocytic growth and tumourigenesis. Pigment Cell Melanoma
Res 21: 611-626.

FELSENSTEIN, J. (1985). Confidence limits on phylogenies: an approach using the bootstrap. Evolution 39: 783-791.

GEORGAS, K., RUMBALLE, B., VALERIUS, M.T., CHIU, H.S., THIAGARAJAN, R.D., LESIEUR, E., ARONOW, B.J., BRUNSKILL, E.W., COMBES, A.N., TANG, D. et al., (2009). Analysis of early nephron patterning reveals a role for distal RV proliferation in fusion to the ureteric tip via a cap mesenchyme-derived connecting segment. Dev Biol 332: 273-286.

HALL, T.A. (1999). BioEdit: a user-friendly biological sequence alignment editor and analysis program for Windows 95/98/NT. Nucl Acids Symp Ser 41: 95-98.

HUELSENBECK, J.P. and RONQUIST, F. (2001). MrBayes: Bayesian inference of phylogeny. Bioinformatics 17: 754-755

KUMAR, S., STECHER, G. and TAMURA, K. (2016). MEGA7: Molecular Evolutionary Genetics Analysis version 7.0 for bigger datasets. Mol Biol Evol 33: 1870-1874.

LE BOUFFANT, R., CUNIN, A.C., BUISSON, I., CARTRY, J., RIOU, J.F. and UMB HAUER, M. (2014). Differential expression of arid5b isoforms in Xenopus laevis pronephros. Int J Dev Biol 58: 363-368.

LOUIS, A., NGUYEN, N.T., MUFFATO, M. and CROLLIUS, H.R. (2015). Genomicus update 2015: KaryoView and MatrixView provide a genome-wide perspective to multispecies comparative genomics. Nucleic Acids Res 43: D682-689.

MUTAI, H., NAGASHIMA, R., SUGITANI, Y., NODA, T., FUJII, M. and MATSUNAGA T. (2009). Expression of Pou3f3/Brn-1 and its genomic methylation in developing auditory epithelium. Dev Neurobiol 69: 913-930.

NAKAI, S., SUGITANI, Y., SATO, H., ITO, S., MIURA, Y., OGAWA, M., NISHI, M. JISHAGE, K., MINOWA, O. and NODA, T. (2003). Crucial roles of Brn1 in dista tubule formation and function in mouse kidney. Development 130: 4751-4759.

NIEUWKOOP, P.D.A.F., J. (1967). Normal table of Xenopus laevis (Daudin). NorthHolland Publishing Company. Amsterdam.

PHIPPARD, D., LU, L., LEE, D., SAUNDERS, J.C. and CRENSHAW, E.B., 3RD. (1999). Targeted mutagenesis of the POU-domain gene Brn4/Pou3f4 causes developmental defects in the inner ear. J Neurosci 19: 5980-5989.

RACITI, D., REGGIANI, L., GEFFERS, L., JIANG, Q., BACCHION, F., SUBRIZI, A.E. CLEMENTS, D., TINDAL, C., DAVIDSON, D.R., KAISSLING, B. et al., (2008). Organization of the pronephric kidney revealed by large-scale gene expression mapping. Genome Biol 9: R84.

RIEGER, A., KEMTER, E., KUMAR, S., POPPER, B., AIGNER, B., WOLF, E., WANKE, R. and BLUTKE, A. (2016). Missense mutation of POU domain class 3 transcription factor 3 in Pou $3{ }^{2} 3^{L 423 P}$ mice causes reduced nephron number and impaired development of the thick ascending limb of the loop of Henle. PLoS One 11: e0158977.

RYAN, A.K. and ROSENFELD, M.G. (1997). POU domain family values: flexibility, partnerships, and developmental codes. Genes Dev 11: 1207-1225.

SCHMIDT, A., SOMMER, F., REINER, M., KLOTZ, T., ENGELMANN, U., ADDICKS, K. and BLOCH, W. (2005). Differential endostatin binding to bladder, prostate and kidney tumour vessels. BJU Int 95: 174-179.

SCHWARZ R. and DAYHOFF M. (1979). Matrices for detecting distant relationships. In Atlas of protein sequences (Ed. M. Dayhoff). National Biomedical Research Foundation, Washington D.C., pp. 353-358.

SMEETON, J., DHIR, P., HU, D., FEENEY, M.M., CHEN, L. and ROSENBLUM, N.D. (2016). Integrin-linked kinase controls renal branching morphogenesis via dual specificity phosphatase 8. J Am Soc Nephrol 27: 1465-1477.

SQUARE, T., JANDZIK, D., CATTELL, M., COE, A., DOHERTY, J. and MEDEIROS D.M. (2015). A gene expression map of the larval Xenopus laevis head reveals developmental changes underlying the evolution of new skeletal elements. Dev Biol 397: 293-304.

VAN DE PEER, Y., MIZRACHI, E. and MARCHAL, K. (2017). The evolutionary significance of polyploidy. Nat Rev Genet 18: 411-424

WITTA, S.E., AGARWAL, V.R. and SATO, S.M. (1995). XIPOU 2, a noggin-inducible gene, has direct neuralizing activity. Development 121: 721-730.

ZHAO, F.Q. (2013). Octamer-binding transcription factors: genomics and functions Front Biosci 18: 1051-1071.

ZHU, Q., SONG, L., PENG, G., SUN, N., CHEN, J., ZHANG, T., SHENG, N., TANG, W., QIAN, C., QIAO, Y. et al., (2014). The transcription factor Pou3f1 promotes neural fate commitment via activation of neural lineage genes and inhibition of external signaling pathways. eLife 3: e02224. 


\section{Further Related Reading, published previously in the Int. J. Dev. Biol.}

Chromatin assembly and transcriptional cross-talk in Xenopus laevis oocyte and egg extracts

Wei-Lin Wang and David Shechter

Int. J. Dev. Biol. (2016) 60: 315-320

https://doi.org/10.1387/ijdb.160161ds

Expressional characterization of mRNA (guanine-7) methyltransferase (rnmt) during early development of Xenopus laevis Ashwin Lokapally, Sanjeeva Metikala and Thomas Hollemann

Int. J. Dev. Biol. (2016) 60: 65-69

https://doi.org/10.1387/ijdb.150409th

pdzrn3 is required for pronephros morphogenesis in Xenopus laevis

Silvia Marracci, Alberto Vangelisti, Vittoria Raffa, Massimiliano Andreazzoli and Luciana Dente

Int. J. Dev. Biol. (2016) 60: 57-63

https://doi.org/10.1387/ijdb.150381ld

Expression of the novel gene Ened during mouse and Xenopus embryonic development Renata Meszaros, Ina Strate, Edgar M. Pera and Madeleine Durbeej

Int. J. Dev. Biol. (2008) 52: 1119-1122

https://doi.org/10.1387/ijdb.082700rm

The role of XTRAP-gamma in Xenopus pronephros development

Dong-hui Li, Techuan Chan, Reiko Satow, Shinji Komazaki, Kouhei Hashizume and Makoto Asashima

Int. J. Dev. Biol. (2005) 49: 401-408

http://www.intjdevbiol.com/web/paper/052005dl

Embryonic expression of Xenopus SGLT-1L, a novel member of the solute carrier family 5 (SLC5), is confined to tubules of the pronephric kidney

Samer R Eid, Anne Terrettaz, Katsumi Nagata and André W Brändli

Int. J. Dev. Biol. (2002) 46: 177-184

http://www.intjdevbiol.com/web/paper/11902681

Towards a molecular anatomy of the Xenopus pronephric kidney

A W Brändli

Int. J. Dev. Biol. (1999) 43: 381-395

http://www.intjdevbiol.com/web/paper/10535314

5 yr ISI Impact Factor $(2016)=2.421$

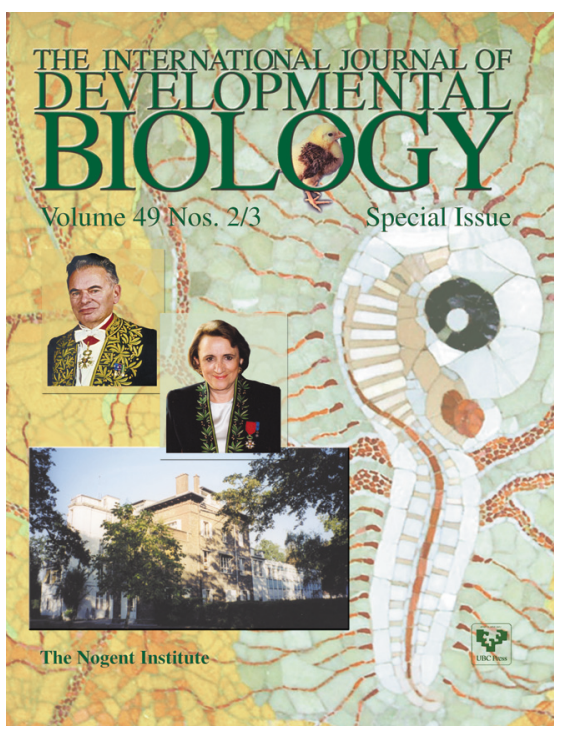

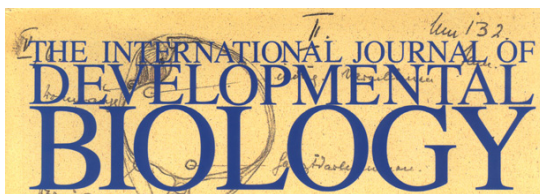

Volume 45 No. 1

Special issue

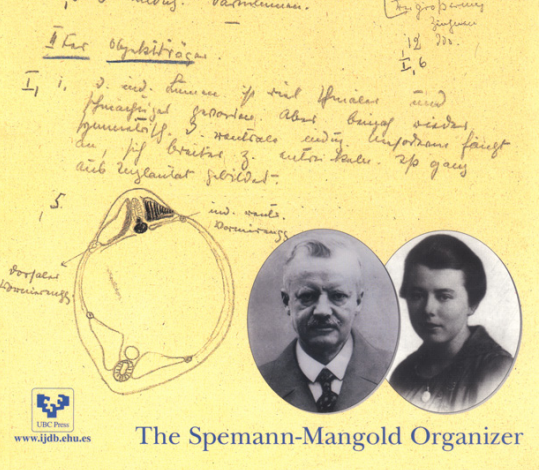

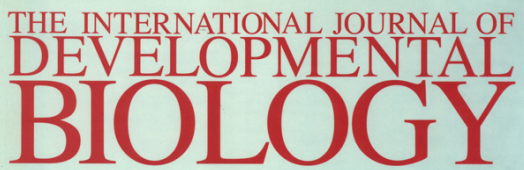
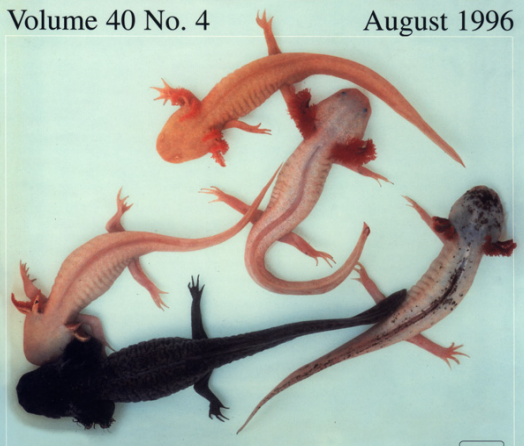

Developmental Biology of Urodeles
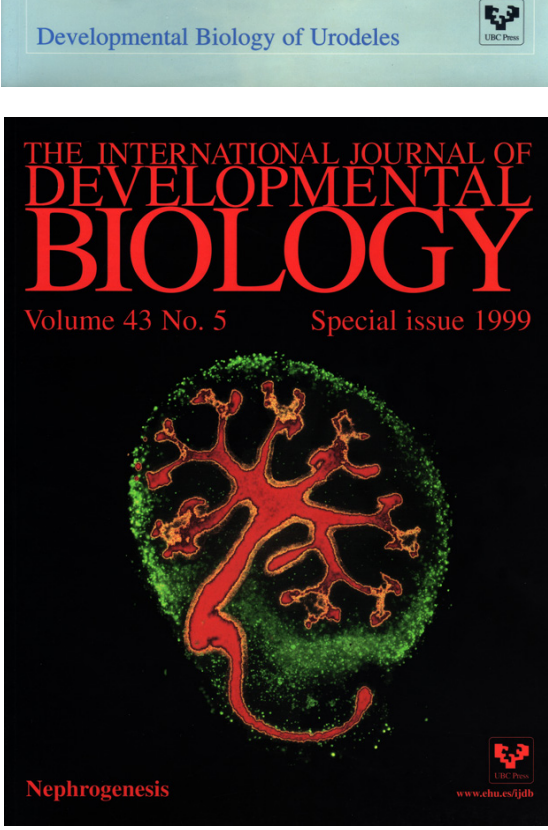